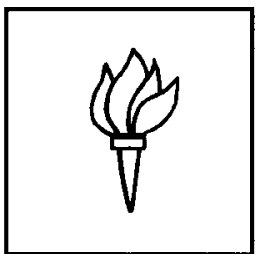

NEW YORK UNIVERSITY

STERN SCHOOL OF BUSINESS

FINANCE DEPARTMENT

Working Paper Series, 1994

Multivariate Binomial Approximations for Asset Prices with Non-Stationary Variance and Covariance Characteristics

Teng-Suan Ho, Richard C. Stapleton, and Marti G. Subrahmanyam

FD-94-36 

Review of Financial studies

Forthcoming

\title{
Multivariate Binomial Approximations for Asset \\ Prices with Non-Stationary Variance \\ and Covariance Characteristics
}

\author{
Teng-Suan Ho \\ Richard C. Stapleton \\ Lancaster University \\ Marti G. Subrahmanyam \\ New York University
}

Earlier versions of this paper have been presented at the European Finance Association Conference, Lisbon, Portugal, Aarhus University, Denmark, Melbourne University, Australia, University of Michigan and Virginia Polytechnic Institute, USA. We are grateful to participants at these presentations and to John Chang, Chi-fu Huang (the editor), Apoorva Koticha, Ser-Huang Poon, Steven Tsay and an anonymous referee for comments on previous drafts. Address correspondence and reprint requests to T.S. Ho, Department of Accounting and Finance, The Management School, Lancaster University, Lancaster LA1 4YX, England, U.K. 



\begin{abstract}
In this paper, we suggest an efficient method of approximating a general, multivariate lognormal distribution by a multivariate binomial process. There are two important features of such multivariate distributions. First, the state variables may have volatilities that change over time. Second, the two or more relevant state variables involved may covary with each other in a specified manner, with a time-varying covariance structure. We discuss the asymptotic properties of the resulting processes and show how the methodology can be used to value a complex, multiple-exerciseable option whose payoff depends on the prices of two assets.
\end{abstract}



In practice, many problems in the valuation of derivative assets are solved by using binomial approximations to continuous distributions. In this paper, we suggest an efficient method of approximating a general, multivariate lognormal distribution by a multivariate binomial process. There are two features of such multivariate lognormal distributions that are of interest. First, the state variables may have volatilities that change over different time intervals, i.e. exhibit a term structure of volatility, either because of changing volatility or mean-reversion. Second, there may be two or more relevant state variables involved, for example a commodity price and a foreign exchange rate, which may covary with each other in a specified manner.

The binomial approach to the valuation and hedging of options has become increasingly important with the creation of new exotic derivative products. Many options with path-dependent payoffs, for example, American options and Asian or average-rate options, may be valued using the binomial methodology ${ }^{1}$. Also, in the analysis of interest rate derivative products, it is often useful to model the construction and evolution of the term structure of interest rates using a binomial process. The present paper provides a general methodology for the construction of binomial approximations of multivariate lognormal distributions, both across state variables and across time.

In order to illustrate the type of problems that can be solved using the methods presented in this paper, consider the following example of an option on oil ${ }^{2}$. The contract is an American-style option which allows the holder to buy oil at a specified price denominated in Japanese yen. The payoff on the option in yen depends upon two variables, the U.S. dollar-denominated oil price and the yen/dollar foreign exchange rate. At time 0 , suppose that the volatility of the oil price is high, but is expected to decline over the life of the option. Also, suppose that there is significant mean-reversion in the price of oil. In contrast, in this example, the yen-dollar exchange rate is assumed to have constant volatility and no mean-reversion. Further, suppose that the correlation between the price of oil and the yen-dollar exchange rate is high, but is expected to fall.

We wish to value this oil option by approximating the true joint distribution of the variables with a bivariate binomial process. The method developed in this paper is designed 
to solve this general problem of changing volatilities, mean-reversion and multiple state-variables, whereas existing models in the literature only deal with one or two aspects of the problem at a time ${ }^{3}$. For example, Nelson and Ramaswamy (1990) show how to approximate various univariate distributions with binomial processes. Amin (1991) derives an alternative approach to the problem of a changing volatility function. However, neither paper deals with changing volatility in a multivariate context. Boyle (1988) and Amin (1991) provide models of the multivariate case, but they deal with either one asset in the context of changing volatility or multiple assets with a constant correlation structure. Amin, for example assumes a constant covariance matrix, when dealing with the multivariate problem explicitly. The incremental contribution of our paper is that it deals with the general multivariate problem, where the assets may have volatilities changing over time but differently across assets, a changing covariance matrix and differential mean reversion.

In the literature, one method suggested to solve both the univariate and multivariate problem involves a change in the conditional probability computed on a node-by-node basis. In this paper, we explicitly recognize that the two problems are really two aspects of the same general issue. In other words, we need, in general, to choose the probabilities so that both the time series (term structure of volatility) and cross-sectional (correlation structure) characteristics of the underlying asset prices are satisfied. We show that an application of the linear multiple regression property of joint-normally distributed variables leads to a formula for the appropriate conditional probabilities. Application of this linear relationship yields a multivariate binomial distribution which is simple to compute and apply.

In Section 1, we introduce the problem in a formal manner and define our notation. In Section 2, we review the literature on the topic of binomial approximation of lognormally distributed variables and relate our work to the previous research. Section 3 then describes the method in the context of a single variable that has both volatility changes and mean reversion. Section 4 derives and demonstrates a similar methodology for the general multivariate case described above. Our conclusions are summarized in Section 5. 


\section{Statement of the Problem and Notation}

We assume that the prices of each of the underlying assets, $X_{1}, X_{2}, \ldots, X_{j}, \ldots, X_{J}$ follows a lognormal diffusion process:

$$
d \ln X_{j}=\mu_{j}\left(X_{j}, t\right) d t+\sigma_{j}(t) d Z_{j}, \quad j=1,2, \ldots, J
$$

where $\mu_{j}$ and $\sigma_{j}$ are the instantaneous drift and volatility of $\ln X_{j}$, and $d Z_{j}$ is a standard Brownian motion. The instantaneous correlation between the Brownian motions, $d Z_{j}$ and $d Z_{k}$ is $\rho_{j, k}(t)$. The instantaneous drift in equation (1) is a function of $X$ and $t$, which allows for mean reversion that may change over time. We assume that $\mu\left(X_{j}, t\right)$ is linear in $X$ and the instantaneous variances and covariances are non-stochastic functions of time. Hence, the asset prices are lognormally distributed at any time $t$. There is a finite number $(m)$ of future dates in the time interval $[0, T]$ at which we are interested in the asset prices. The dates are numbered $t_{1}, t_{2}, \ldots, t_{i}, \ldots, t_{m}$, where $t_{m}=T$ and these are determined by the requirements of the option valuation problem that is being solved. ${ }^{4} \mathrm{We}$ are interested in the joint distribution of the prices of the assets on these dates.

We denote the unconditional mean (at time 0 ) of the logarithmic asset return at time $i$ as $\mu_{i, j}$. The conditional volatility over the period $i-1$ to $i$ is denoted $\sigma_{i-1, i, j}$, and the unconditional volatility is $\sigma_{0, i, j}$. Also, the covariances across assets at time $t_{i}$ are respectively

$$
\begin{aligned}
\operatorname{cov}_{0}\left(\ln X_{i, j}, \ln X_{i, k}\right) & =\rho_{0, i, j, k} \sigma_{0, i, j} \sigma_{0, i, k} \equiv \sigma_{0, i, j, k}, \\
\operatorname{cov}_{i-1}\left(\ln X_{i, j}, \ln X_{i, k}\right) & =\rho_{i-1, i, j, k} \sigma_{i-1, i, j} \sigma_{i-1, i, k} \equiv \sigma_{i-j, i, j, k},
\end{aligned}
$$

where the correlation coefficients, $\rho_{0, i, j, k}$ and $\rho_{i-1, i, j, k}$ are specified exogenously. ${ }^{5}$

The conditional and unconditional volatilities depend, in general, on the functional form of $\mu_{j}\left(X_{j}, t\right)$ and $\sigma_{j}(t)$. For example, if $\mu_{j}$ is a constant, $\ln X_{j}$ follows a random walk with

$$
\sigma_{0, i, j}=\sqrt{\frac{1}{t_{i}} \int_{0}^{t_{i}} \sigma_{j}^{2}(t) d t}
$$


and

$$
\sigma_{i-1, i, j}=\sqrt{\frac{1}{t_{i}-t_{i-1}} \int_{i_{i-1}}^{t_{i}} \sigma_{j}^{2}(t) d t} .
$$

In general, however, the volatilities $\sigma_{0, i, j}$ and $\sigma_{i-1, i, j}$ are complex functions of the instantaneous volatilities $\sigma_{j}(t)$. In this paper, instead of starting with a particular specified process for $X_{j}$, we assume, more generally that the relevant means, volatilities and conditional volatilities are given exogenously. ${ }^{6}$

We wish to approximate the process in equation (1) with a sequence of binomial distributions such that the mean, variance and covariance characteristics converge to these given values. The sequence of binomial distributions yields vectors of outcomes of $X_{i, j}$, where $X_{i, j}$ is the price of asset $j$ at time $t_{i}, i=1,2, \ldots, m$. Conditional probabilities have to be so chosen that the volatility specifications and correlations are satisfied for each time period.

Since we are concerned only with a finite set of dates, $m$, the data input required consists of $m$ vectors of exogenously given means $\left(\bar{\mu}_{1}, \bar{\mu}_{2}, \ldots, \bar{\mu}_{i}, \ldots, \bar{\mu}_{m}\right)$ where $\bar{\mu}_{i}=\left(\mu_{i, 1}, \ldots, \mu_{i, J}\right)$, and $m$ unconditional covariance matrices $\left(\Sigma_{0,1}, \Sigma_{0,2}, \ldots, \Sigma_{0, m}\right)$, where ${ }^{7}$

$$
\Sigma_{0, i}=\left[\begin{array}{ccccc}
\sigma_{0, i, 1}^{2} & \ldots & \sigma_{0, i, 1, k} & \ldots & \sigma_{0, i, 1, J} \\
& \ldots & \ldots & \ldots & \ldots \\
& & \sigma_{0, i, k}^{2} & \ldots & \sigma_{0, i, k, J} \\
& & & \ldots & \ldots \\
& & & & \sigma_{0, i, J}^{2}
\end{array}\right]
$$

and $m-1$ conditional covariance matrices $\left(\Sigma_{1,2}, \Sigma_{2,3}, \ldots, \Sigma_{m-1, m}\right)$ where:

$$
\sum_{i-1, i}=\left[\begin{array}{ccccc}
\sigma_{i-1, i, 1}^{2} & \ldots & \sigma_{i-1, i, 1, k} & \ldots & \sigma_{i-1, i, 1, J} \\
& \ldots & \ldots & \ldots & \ldots \\
& & \sigma_{i-1, i, k}^{2} & \ldots & \sigma_{i-1, i, k, J} \\
& & & \ldots & \ldots \\
& & & & \sigma_{i-1, i, J}^{2}
\end{array}\right] .
$$

The exogenously given drift and volatility terms are satisfied asymptotically by choosing the number of binomial stages between 0 and $t_{1}, t_{1}$ and $t_{2}$, etc.. There are $n_{1}$ binomial stages between 0 and $t_{1}, n_{2}$ stages between $t_{1}$ and $t_{2}$, and so on. As we discuss 
below, the ability to choose the "denseness" of the binomial tree between any two dates represents a significant difference between our model and the previous binomial approximations available in the literature.

In our method, we first construct a binomial approximation for each of the state variables on which the option payoff depends. We then model the given covariance structure by adjusting the conditional probabilities of $X_{i, k}$ given the outcomes of $X_{i, j} \cdot{ }^{8}$ Since our methodology is closely related to, but significantly different from the previous work cited above, we now discuss the contribution of these papers.

\section{Relationship to the Literature on Binomial Approxi- mations and Option Pricing}

The method we use to approximate the multivariate process is closely related to previous contributions by Boyle (1988), Nelson and Ramaswamy (1990) [NR], Amin (1990, 1991), Amin and Bodurtha (1994) and He (1990). ${ }^{9}$ The basic idea in NR is to approximate a given univariate process for the price for the underlying asset by a "simple" binomial process. In the NR context, "simple" means that the number of nodes of the binomial process increases linearly with time. In order to ensure that the process has the desired variance characteristics, while remaining simple, NR suggest an adjustment in the conditional probabilities of the binomial process over time.

In this paper, we also construct simple binomial processes. However, in contrast to $\mathrm{NR}$, we allow the number of binomial stages $n_{i}$ between any two points $t_{i-1}$ and $t_{i}$ to be greater than 1. This means that, in the univariate case, the NR method can be regarded as a special case of ours, where $t_{i}-t_{i-1}$ is the same for all $i$ and also $n_{i}=1$, for all $i$. In our approach, even in the multivariate case, we can, if we wish, accommodate a change in volatility by allowing $n_{i}$ to vary. This follows from a suggestion of Amin (1991), but with an important difference. We do not need to change the time intervals, $\left(t_{i-1}, t_{i}\right)$, in our more general setting. This means that our method is readily extendible to the multivariate case. 
It is well known that a multivariate process across two or more assets can also be modelled by changing the conditional probabilities associated with the nodes. Boyle (1988) shows how to construct a trinomial distribution for each variable and then combine them in a lattice, so that the variables have the required variance and covariance characteristics. Amin (1991) models the covariance characteristics of the assets assuming a constant variance-covariance matrix, i.e. one where the asset prices have constant volatilities over time. He then compares the efficiency of Boyle's method against a range of alternatives, including two where the variables are joint-binomially distributed. He finds that, from the standpoint of computational efficiency, the latter approaches are marginally inferior. However, if the factors generating the asset prices ("shocks" in his Example 2) are chosen so as to be independent, the binomial model has the advantage that it does not suffer from the problem of "negative probabilities"' that arises in the Boyle approach. He (1990) and Amin (1990) use a trinomial distribution to model a bivariate distribution of two factors. They do it in such a way that the factors are uncorrelated, but not independent. He (1990) shows that the distribution converges to the multivariate Brownian motion assumed and that option prices also converge. These models do not incorporate changing conditional variances or mean reversion.

In our model, we choose the multivariate binomial method as suggested by Amin's work. The major difference between our model and the work by Amin and Boyle is that unlike their approaches, the $n_{i}$ in our case is not constrained to be 1 through time. We are then able to model simultaneously the effects of both changing volatility and mean-reversion in the case of two or more underlying assets.

In summary, we extend the existing literature in two directions. First, in the univariate case, we are able to extend the NR and Amin models by allowing the number of binomial stages to exceed 1 and to vary within the time intervals, $\left(t_{i-1}, t_{i}\right)$. This means that we can control the "denseness" of the binomial tree between the multiple exercise dates of the option. Our technique can then be used, for instance, to extend Breen's (1991) accelerated binomial option pricing model to the case where volatility changes and/or the asset price 
mean-reverts. Secondly, we are able to extend Amin's work by modelling a general multivariate process, where the individual assets have different rates of change of variance and mean-reversion.

\section{A Method for Constructing a Univariate Binomial Process with Specified Variances}

The problem, in the univariate case, is to approximate with a binomial process the true process for $X_{i}$, given the means, $\mu_{i}$, conditional volatilities, $\sigma_{i-1, i}$, and the unconditional volatilities, $\sigma_{0, i}{ }^{10}$ The conditional volatilities of the approximated binomial process will be denoted $\hat{\sigma}_{i-1, i}\left(n_{i}\right)$ since they will be a functions of $n_{i}$, the number of binomial stages. We require that

$$
\lim _{n_{i} \rightarrow \infty} \hat{\sigma}_{i-1, i}\left(n_{i}\right)=\sigma_{i-1, i}, \quad \forall i
$$

The unconditional volatility of the approximated process over the period $\left(0, t_{i}\right)$ is similarly denoted $\hat{\sigma}_{0, i}\left(n_{1}, n_{2}, \ldots, n_{i}\right)$, since it is, in general, a function of the number of binomial stages over each of the sub-periods $t_{1}, t_{2}, \ldots, t_{i}$. Here, we require

$$
\lim _{n_{i} \rightarrow \infty} \hat{\sigma}_{0, i}\left(n_{1}, n_{2}, \ldots, n_{i}\right)=\sigma_{0, i}, \quad \forall i, l, \quad l=1, \ldots, i \text {. }
$$

In addition, of course, we constrain the mean of the approximated process to be equal to $\mu_{i}$ for each $i$. In other words

$$
\lim _{n_{i} \rightarrow \infty} \hat{\mu}_{i}=\mu_{i}, \quad \forall i
$$

Our method involves the construction of $m$ separate binomial distributions, where the time periods are denoted $t_{1}, t_{2}, \ldots, t_{i}, \ldots, t_{m}$. The set of these distributions forms a discrete stochastic process for $X_{i}$ :

$$
\left(X_{1}, X_{2}, \ldots, X_{m}\right)
$$

where $X_{i}$ is only defined at the times $t_{i}$. An example, where $m=2$ is shown in Figure 1 . $X_{1}$ is an $N_{1}+1$ vector where at node $r$,

$$
X_{1, r}=X_{0} u_{1}^{N_{1}-r} d_{1}^{r},
$$


where $N_{1}=n_{1} . X_{2}$ is an $N_{2}+1$ dimensioned vector with

$$
X_{2, r}=X_{0} u_{2}^{N_{2}-r} d_{2}^{r}
$$

at node $r$, where $N_{2}=n_{1}+n_{2}$. We have to choose the up and down movements $u_{1}, u_{2}, d_{1}$ and $d_{2}$ and the probabilities so that equations (4), (5) and (6) are satisfied. We denote

$$
x_{i}=\ln \left(X_{i} / X_{0}\right)
$$

and the conditional probabilities of $x_{2}$ given a node $r$ at $t_{1}$ as

$$
q\left(x_{2} \mid x_{1}=x_{1, r}\right)
$$

or $q\left(x_{2}\right)$ for short.

In general, we need to choose values for the $u_{i}, d_{i}$ and $q\left(x_{2}\right)$ so that the convergence equations (4), (5) and (6) are satisfied. We first establish a lemma which guarantees that the conditional volatility and the unconditional mean converge to their values in (4) and (6).

Lemma 1. Suppose that the up and down movements $u_{i}$ and $d_{i}$ are chosen so that

$$
\begin{aligned}
& d_{i}=\frac{2\left(E\left(X_{i}\right) / X_{0}\right)^{\frac{1}{N_{i}}}}{1+\exp \left(2 \sigma_{i-1, i} \sqrt{\left(t_{i}-t_{i-1}\right) / n_{i}}\right)}, \quad i=1,2, \ldots, m \\
& u_{i}=2\left(E\left(X_{i}\right) / X_{0}\right)^{\frac{1}{N_{i}}}-d_{i}, \quad i=1,2, \ldots, m \\
& N_{i}=\sum_{l=1}^{i} n_{l},
\end{aligned}
$$

where

then if, for all $i$, the conditional probability $q\left(x_{l}\right) \rightarrow 0.5$ as $n_{l} \rightarrow \infty$, for $l=1,2, \ldots, i$, then the unconditional mean and the conditional volatility of the of the approximated process approach respectively their true values:

$$
\lim _{\substack{n_{i} \rightarrow \infty \\ l=1,2, \ldots, i}} \frac{\hat{E}\left(X_{i}\right)}{X_{0}} \rightarrow \frac{E\left(X_{i}\right)}{X_{0}}, \quad \lim _{n_{i} \rightarrow \infty} \hat{\sigma}_{i-1, i} \rightarrow \sigma_{i-1, i} .
$$

Proof. See Appendix A. 
The up and down movements, $u_{i}$ and $d_{i}$ are analogous to those in Cox, Ross and Rubinstein (1979), in that they are chosen to match the true mean and conditional volatility. Of course, in our case since the conditional volatilities are allowed to change over time, the $u_{i}$ 's and $d_{i}$ 's also change correspondingly.

Given Lemma 1, we can guarantee that the mean and conditional volatility of the approximated process converge to their required values, by choosing $u_{i}$ and $d_{i}$ using the given conditional volatilities $\sigma_{i-1, i^{*}}{ }^{11}$ The remaining problem is to choose the conditional probabilities $q\left(x_{i}\right)$ in such a manner that the unconditional volatility converges to the true value as in equation (5). First note that, by Lemma 1, we are free to choose $q\left(x_{i}\right)$ without affecting the convergence of the conditional volatility, as long as $q\left(x_{i}\right) \rightarrow 0.5$ as $n_{i} \rightarrow \infty$. Thus we can choose the probabilities so that both the unconditional and conditional volatilities converge.

Since $x_{i}=\ln \left(X_{i} / X_{0}\right)$ is normally distributed, it follows that the regression

$$
x_{i}=a_{i}+b_{i} x_{i-1}+\varepsilon_{i}, \quad E_{i-1}\left(\varepsilon_{i}\right)=0
$$

is linear with

$$
b_{i}=\sqrt{\left[t_{i} \sigma_{0, i}^{2}-\left(t_{i}-t_{i-1}\right) \sigma_{i-1, i}^{2}\right] / t_{i-1} \sigma_{0, i-1}^{2}}
$$

and $^{12}$

$$
a_{i}=E\left(x_{i}\right)-b_{i} E\left(x_{i-1}\right) .
$$

We now choose the conditional probabilities $q\left(x_{i}\right)$ so that

$$
E_{i-1}\left(x_{i}\right)=a_{i}+b_{i} x_{i-1}
$$

also holds for the approximated variables $x_{i}$ and $x_{i-1} \cdot{ }^{13}$ In Appendix B we show how to choose the conditional probability $q\left(x_{i}\right)$ so as to guarantee that (9) holds. We can now establish: 
Theorem 1. Suppose that the $X_{i}$ are joint lognormally distributed. If the $X_{i}$ are approximated with binomial distributions with $N_{i}=N_{i-1}+n_{i}$ stages and $u_{i}$ and $d_{i}$ given by (7) and (8), and if the conditional probability of an up-movement at noder at time $i-1$ is

$$
q\left(x_{i} \mid x_{i-1}=x_{i-1, r}\right)=\frac{a_{i}+b_{i} x_{i-1, r}-\left(N_{i-1}-r\right) \ln u_{i}-r \ln d_{i}}{n_{i}\left(\ln u_{i}-\ln d_{i}\right)}-\frac{\ln d_{i}}{\ln u_{i}-\ln d_{i}}, \quad \forall i, r
$$

then $\hat{\mu}_{i} \rightarrow \mu_{i}$ and $\hat{\sigma}_{0, i} \rightarrow \sigma_{0, i}$ and $\hat{\sigma}_{i-1, i} \rightarrow \sigma_{i-1, i}$ as $n_{i} \rightarrow \infty, \forall i$.

Proof. First note that, as $n_{i} \rightarrow \infty$

$$
q\left(x_{i}\right) \rightarrow \frac{-\ln d_{i}}{\ln u_{i}-\ln d_{i}} \rightarrow 0.5 .
$$

Hence the conditions of Lemma 1 are fulfilled and $\hat{\sigma}_{i-1, i}$ converges to $\sigma_{i-1, i}$.

We now prove the convergence of $\hat{\sigma}_{0, i} \rightarrow \sigma_{0, i}$ by induction. First, we choose for $i=1, q\left(x_{1}\right)=0.5$. Hence from Lemma 1 the theorem is true for $i=1$. We need now to show that $\hat{\sigma}_{0,2} \rightarrow \sigma_{0,2}$ as $n_{1}$ and $n_{2} \rightarrow \infty$. Then, it follows by induction, that $\hat{\sigma}_{0, i} \rightarrow \sigma_{0, i}$, for all $i$. Lemma 1 guarantees that $\hat{\sigma}_{1,2} \rightarrow \sigma_{1,2}$ if $q\left(x_{2}\right)$ is chosen by equation (14). In Appendix B we show that, with this choice of $q\left(x_{2}\right)$ then $^{14}$

$$
\underset{t_{1}}{\hat{E}}\left(x_{2}\right)=a_{2}+b_{2} x_{1} \text {. }
$$

The volatility $\hat{\sigma}_{0,2}$ is therefore

$$
\hat{\sigma}_{0,2}=\sqrt{\left[b_{2}^{2} \hat{\sigma}_{0,1}^{2} t_{1}+\left(t_{2}-t_{1}\right) \hat{\sigma}_{1,2}^{2}\right] / t_{2}} .
$$

Now, since $\hat{\sigma}_{0,1}=\sigma_{0,1}$ and $\hat{\sigma}_{1,2} \rightarrow \sigma_{1,2}$ as $n_{2} \rightarrow \infty$, then

$$
\hat{\sigma}_{0,2} \rightarrow \sqrt{\left[b_{2}^{2} \sigma_{0,1}^{2} t_{1}+\left(t_{2}-t_{1}\right) \sigma_{1,2}^{2}\right] / t_{2}}
$$

and substituting

$$
b_{2}^{2}=\left[t_{2} \sigma_{0,2}^{2}-\left(t_{2}-t_{1}\right) \sigma_{1,2}^{2}\right] / t_{1} \sigma_{0,1}^{2}
$$

we find that $\hat{\sigma}_{0,2} \rightarrow \sigma_{0,2}$. It follows that if $n_{i} \rightarrow \infty, \quad \hat{\sigma}_{0, i} \rightarrow \sigma_{0, i}, \forall i$. 


\section{Properties of the Approximation for small $n$}

In practical applications we are forced to approximate the stochastic process of $X_{t}$ using small values of $n_{i}$. The smaller the $n$, the more rapid will be the computation of option prices. When $n_{i}$ is small, the conditional probability at a node suggested by the formula in equation (10) could be outside the natural bounds for probability, i.e. $q$ could exceed 1 or be less than $0 .{ }^{15}$

In Table 1, we show the effect of the restriction $0 \leq q\left(x_{i} \mid x_{i-1}=x_{i-1, r}\right) \leq 1, \forall i, r$. We simulate the model for three cases. In the first case, the process for $X$ has the mean reversion property. In the second case, the process for $X_{t}$ exhibits declining conditional volatility. Finally, the third case combines the mean reversion property of Case 1 and the changing volatility of Case 2 .

The simulations all show, as we would expect, that the accuracy of the approximation improves as $n$ increases. The first panel indicates that, for a constant variance process, mean reversion is easily captured by the method, even for relatively small $n$. This is also true for cases of declining volatility in panel 2. The method is accurate for quite small $n$ size and converges rapidly for reasonable changes in volatility. When the volatility declines more rapidly from $10 \%$ in the first period to $7 \%$ in the second, then the accuracy begins to decline somewhat. This is due to the reliance of the method on the conditional volatility for the calculation of $X_{2}$. In extreme cases, however, a change in the size of $n_{i}$ can be made which can improve the accuracy. ${ }^{16}$

Theorem 1 allows us to approximate a process with given mean, variance and covariance characteristics over the periods $\left(0, t_{i}\right)$ and $\left(t_{i-1}, t_{i}\right)$, where $i=1,2, \ldots, m$. We can therefore construct a process using all the dates $t_{1}, t_{2}, \ldots, t_{m}$. Successive application of Theorem 1 guarantees that the volatilities converge to their given values over each time period. The resulting binomial process can be used to evaluate multiple exerciseable options with a given number of exercise dates as in Breen (1991). 


\section{The Multivariate Case}

There are two ways in which an option valuation problem may involve a multivariate probability distribution. The first is when that the payoff on the option depends on the outcome of two or more random variables. This is the case for the yen oil option discussed in the introduction. The payoff there depends upon the dollar oil price and the yen/dollar exchange rate. The second motivation stems from the consideration of the value of an American style option where the intermediate value of the option depends upon the price of the underlying asset and other variables, such as the rate of interest. In this case we need to model the covariance of the asset price and the rate of interest as well as the time series properties of the two variables. In this Section we consider the general problem of approximating the multivariate distribution of two or more variables each of which has the properties (of mean reversion and changing volatility) discussed above in Section 3. ${ }^{17}$

Initially, we consider the case of a single time period $(0, T)$. This is relevant for European style options where the payoff depends upon two or more variables. We wish to approximate the joint distribution of the variables $(X, Y, Z, \ldots)$ with a multivariate binomial distribution. Hence, we choose the conditional probabilities (of $Y$ given $X$, and of $Z$ given $Y$ and $X$ ) given the covariance between the variables. ${ }^{18}$ We confine ourselves to the case where there are three relevant random variables $(X, Y, Z)$. The first step involves approximating the first random variable $X_{T}$ with a binomial distribution using the method outlined in Section 3. We then build a vector of $Y_{T}$ values using equations (7) and (8), and using the conditional volatility of $Y_{T}$ given $X_{T}$. We then choose the conditional probability of $Y_{T}$ given the outcome $X_{T}$. This conditional probability is denoted $q\left(y_{T} \mid x_{T}=x_{T, r}\right)$, where $r$ is the node of the $X$ binomial tree, and $x_{T, r}$ is the value of $x_{T}$ at the $r$ th node. In Appendix B we show that the appropriate value of $q\left(y_{T}\right)$ is ${ }^{19}$

$$
q\left(y_{T} \mid x_{T}=x_{T, r}\right)=\frac{\alpha_{y}+\beta_{y} x_{T, r}-n_{y} \ln d_{y}}{n_{y}\left(\ln u_{y}-\ln d_{y}\right)},
$$

where $\alpha_{y}$ and $\beta_{y}$ are the coefficients from the simple regression of $Y_{T}$ on $X_{T}$, and $n_{x}$ and $n_{y}$ are the number of binomial stages in the $x$ and $y$ approximations respectively. 
In the next stage, we build a vector of $Z_{T}$ using the conditional volatility of $Z_{T}$ given $X_{T}$ and $Y_{T}$. We then choose the probability of $Z$ given an $X$ and a $Y$ node. This conditional probability is shown in Appendix B (equation (B3)) to be

$$
q\left(z_{T} \mid x_{T}=x_{T, r}, y_{T}=y_{T, s}\right)=\frac{\alpha_{z}+\beta_{z} x_{T, r}+\gamma_{z} y_{T, s}-n_{z} \ln d_{z}}{n_{z}\left(\ln u_{z}-\ln d_{z}\right)},
$$

where $\alpha_{z}, \beta_{z}$, and $\gamma_{z}$ are the coefficients from the multiple regression of $Z_{T}$ on $X_{T}$ and $Y_{T}$. If the conditional probabilities are chosen by (11) and (12), it follows by an extension of Theorem 1 , that the volatilities of $Y$ and $Z$ converge, as $n_{y}$ and $n_{z}$ increase, to their values $\sigma_{y}$ and $\sigma_{z}$.

We now consider the accuracy of the covariance and correlation between the approximated $X$ and $Y$ variables. We have:

Theorem 2. Suppose the up and down movements of two correlated random variables, $X$ and $Y$, are chosen by equations (7) and (8) and that the conditional probabilities are given by equation (11). Then the approximated value of the conditional covariance is equal to its true value. Also, when there are three or more correlated variables, for example $X, Y$, and $Z$, where $X$ is the first fitted variable, the covariance of the pairs $(X, Y)$ and $(X, Z)$ are exact and that of $(Y, Z)$ limits to its true value.

Proof. See Appendix A.

Note that, in the case of just two variables $X$ and $Y$, this cross-sectional property is an exact one and holds for all values of $n$ and not just for the limiting values. The approximation in the case of three or more variables is due to the fact that $Y$ has only an approximately correct variance which limits to its true value as $n$ increases. Also, the covariance property holds in the multiperiod case for all the conditional covariances. However, since our method approximates the variances of the variables, the resulting correlation is only asymptotically correct. 


\section{The Multiperiod, Multivariate Case}

We now turn to the general problem of a multiperiod, multivariate process. In this case we may be concerned with say two variables (the dollar price of oil and the yen/dollar exchange rate) at a series of dates $\left(t_{1}, t_{2}, \ldots,\right)$. Our general problem is to construct a multiperiod, multivariate process over periods $\left(0, t_{1}\right),\left(t_{1}, t_{2}\right), \ldots$. For simplicity, we illustrate the general methodology with an example of a two period $\left[\left(0, t_{1}\right),\left(t_{1}, t_{2}\right)\right]$, bivariate $(X, Y)$ case. This case is sufficient, for example, if we wish to value a compound option whose payoff depends upon two variables.

Suppose that $\left(X_{1}, X_{2}\right)$ and $\left(Y_{1}, Y_{2}\right)$ are multivariate lognormally distributed with volatilities $\left(\sigma_{0,1, x}, \sigma_{0,2, x}, \sigma_{1,2, x}\right)$ and $\left(\sigma_{0,1, y}, \sigma_{0,2, y}, \sigma_{1,2, y}\right)$. Also, assume that the correlation between $x_{1}$ and $y_{1}$ is $\rho_{0,1, x, y}$. The conditional correlation between $x_{2}$ and $y_{2}$ is denoted $\rho_{1,2, x, y}$. Note that, for the joint lognormal distribution, the conditional correlation is non-stochastic. However, in this method there is no need to restrict the conditional correlation to be the same at each point in time.

The steps in the computation for the general case are:

1. First, compute the up and down movements for $X_{1}$ and $Y_{1}$, and $X_{2}$ and $Y_{2}$ independently using the methodology of Section 3. Specifically, the vector of $Y_{1}$ is constructed using the conditional volatility of $Y_{1}$ given $X_{1}, X_{2}$ requires the conditional volatility $\sigma_{1,2, x}$, and $Y_{2}$ requires the conditional volatility of $Y_{2}$ given both $Y_{1}$ and $X_{2}$.

2. Next, compute the conditional probability of an up movement in $Y_{1}$ given a value of $X_{1}$ using equation (11) above. We denote these probabilities as $q\left(y_{1} \mid x_{1}=x_{1, r}\right)$ since they are of $Y_{1}$ given that $X_{1}$ is at a node $r$ at time $t_{1}$.

3. We then compute the conditional probability of an up movement in $X_{2}$ given a value of $X_{1}$ using the methodology of Section 3 and equation (10) in particular. These probabilities are denoted $q\left(x_{2} \mid x_{1}=x_{1, r}\right)$.

4. Finally, we need to compute the conditional probability of $Y_{2}$ given both $Y_{1}$ and $X_{2}$. We denote this probability as $q\left(y_{2} \mid y_{1}=y_{1, r}, x_{2}=x_{2, s}\right)$. This probability must satisfy both the time series $\left(Y_{2}\right.$ on $\left.Y_{1}\right)$ and cross-sectional $\left(Y_{2}\right.$ on $\left.X_{2}\right)$ properties of $Y_{2}$. In other 
words, it must satisfy the volatilities $\left(\sigma_{0,1, y}, \sigma_{0,2, y}, \sigma_{1,2, y}\right)$ and the conditional correlation $\rho_{1,2, x, y}$. In Appendix $B$, we show that in this case

$$
q\left(y_{2}\right)=\frac{a_{2}+b_{2} y_{1, r}+c_{2} x_{2, r}-\left[\left(N_{1}-r\right) \ln u_{2, y}+r \ln d_{2, y}\right]-n_{2} \ln d_{2, y}}{n_{2}\left[\ln u_{2, y}-\ln d_{2, y}\right]},
$$

where the probability $q\left(y_{2}\right)=q\left(y_{2} \mid y_{1}=y_{1, r}, x_{2}=x_{2, s}\right)$, and where $a_{2}, b_{2}$, and $c_{2}$ are the multiple regression coefficients from the regression of $y_{2}$ on $y_{1}$ and $x_{2}$.

Again, an extension of Theorem 1 can be used to show that when $q\left(y_{2}\right)$ and $q\left(x_{2}\right)$ are chosen in this manner, both the variances and the correlations of the multivariate process converge to their given values. However, the probabilities in equations (10), (11) and (13) could be greater than one or less than zero when the $n$-size is finite. As we see now, this is particularly likely when the correlation between the variables is high.

It is important now to consider the limitations on the accuracy of the multivariate method when the natural limits are placed on the conditional probabilities in (10), (11) and (13). In Table 2, we show the results of simulations with and without a non-negativity constraint placed on the conditional probabilities. For simplicity, we show just the case of a single time period $\left(0, t_{1}\right)$, with $t_{1}=1$ year. Even when the conditional probability is constrained, the simulations show that the accuracy of the approximation is not adversely affected by the non-negativity constraint for correlations under 0.8 . For high values of $\rho$ (for example $\rho=0.9$ ) some inaccuracy is apparent but this reduces for large $n$ values. ${ }^{20}$

\section{Applications of the Methodology to Option Pricing}

In applying the methodology to the pricing of options we need to approximate the risk neutral distribution of the asset prices. For the problems considered here, this distribution is joint lognormal where the means of the variables are their respective forward prices and the volatilities are the same as those of the actual distribution. It follows from Theorem 1 that if the $u_{i}$ and $d_{i}$ are chosen as in equations (7) and (8) with the additional requirement that the mean equals the forward price, then the distribution converges to the required risk neutral distribution. In this Section, we price multiple exerciseable options 
on two variables using these approximating distributions. However, before applying the method we need to establish that the option payoffs and prices also converge as the $n$-size increases.

In the case of a finite $n$-size, we propose the option payoffs and values only as approximations to their limiting values. The method makes no use of the (unknown) true mean of the underlying asset and does not assume a complete market in which no-arbitrage option values are determined. We show that the option values we compute converge to the prices in the complete market, continuous-time economy. ${ }^{21}$

We will first discuss and prove convergence in the case of a European-style call option on a single asset: the Black-Scholes case. We then extend the proof to the case of American-style options. Our procedure in the univariate case is as follows. If the option has a maturity $t$, the asset price on which the option is written has a lognormally distributed price $X_{t}$ with volatility $\sigma$. We construct a binomially distributed variable $X_{t}(n)$ with binomial probability $q=0.5$, an expected value $E\left[X_{t}(n)\right]=F_{0, t}:$ the asset forward price, and $u$ and $d$ chosen by the formula in equations (7) and (8). It follows from Lemma 1 that $X_{t}(n)$ converges in distribution to the risk neutral density of $X_{t}$ : i.e. to a distribution with a mean $F_{0, t}$ and volatility $\sigma$. We now establish

Theorem 3. Define $X_{t}(n)$ using equations (7) and $(8)$ with $E\left[X_{t}(n)\right]=F_{0, t}$. Then

(a) $X_{t}(n)$ converges in distribution to $X_{t}$, where $X_{t}$ is lognormal with mean $F_{0, t}$ and logarithmic variance $\sigma^{2} t$.

(b) For a European-style call option with payoff function $g\left(X_{t}\right)=\max \left[X_{t}-K, 0\right]$, $g\left[X_{t}(n)\right] \rightarrow g\left(X_{t}\right)$.

(c) $E\left[g\left(X_{t}(n)\right)\right] \rightarrow E\left[g\left(X_{t}\right)\right]=C_{0} / B_{0, t}$, where $C_{0}$ is the Black and Scholes value of the call option.

Proof.

(a) Follows directly from Lemma 1.

(b) Follows since $g($.$) is a continuous function, and Billingsley (1986), Theorem$ 25.7, Corollary 1. 
(c) Follows from the fact that the sequence $g\left[X_{t}(n)\right]$ is uniformly integral, given the lognormal distribution, and Billingsley (1986), Theorem 25.12.

Parts (b) and (c) of Theorem 3 follow quite closely the analysis of Duffie (1988, pp. 244-248). An important implication of the theorem is that hedge ratios and other risk parameters such as the deltas, thetas, vegas, and gammas, (i.e. the derivatives of $C_{0}$ ) also converge as the $n$-size increases. This allows the approximation of prices and hedge ratios with the binomial method.

\section{American Style Options}

We now extend the discussion of convergence to the case of American-style options. Consider first a call option that is exerciseable twice at time $t_{1}$ and at time $t_{2}$. Let the exercise price at time $t_{1}$ be $K_{1}$, and at time $t_{2}$ be $K_{2}$. The payoff at time $t$, if the option is not exercised at $t_{1}$ is

$$
g\left(X_{2}\right)=\max \left[X_{2}-K_{2}, 0\right]
$$

The value of the option, if alive at time $t_{1}$, is given by the Black-Scholes function

$$
C_{1}=C_{1}\left(X_{1}\right)
$$

Just prior to the exercise decision at time $t_{1}$, the value of the option can therefore be written

$$
g\left(X_{1}\right)=\max \left[C_{1}, X_{1}-K_{1}\right]
$$

The value at time 0 of the claim is

$$
C_{0}=B_{0,1} E\left[g\left(X_{1}\right)\right]
$$

where the expectation is again taken with respect to the risk neutral density. ${ }^{22}$ We now approximate $C_{0}$ using a binomial distribution for $X_{1}$ and $X_{2}$.

To approximate $C_{0}$, we first construct $X_{1}(n)$ and $X_{2}(n)$ using equations (7) and (8) with $E\left(X_{1}\right)=F_{0,1}$ and $E\left(X_{2}\right)=F_{1,2}$. We then compute $g\left(X_{2}(n)\right)$ and $C_{1}(n)$ in the same way as in the case of the European-style option above. It then follows: 
Corollary 3. The payoff and value of a twice exerciseable option converge to their continuous limits as $n$ increases.

Proof. From Theorem 3, it follows that $C_{1}(n)$ converges to the Black-Scholes value $C_{1}$. We know also that the payoff (if exercised) at $t_{1}, X_{1}(n) \rightarrow X_{1}$. Hence $g\left(X_{1}(n)\right) \rightarrow g\left(X_{1}\right)$. Finally, since $g\left(X_{1}\right)$ is again uniformly integral it follows that the sequence $E\left[g\left(X_{1}(n)\right)\right]$ converges to $E\left[g\left(X_{1}\right)\right]$, and $C_{0}(n)$ converges to $C_{0}$.

The above extension of Theorem 3 shows that our approximation converges for a twice exerciseable option. By induction it must follow that an $m$-times exerciseable option can be approximated in a similar way choosing the $u$ and $d$ proportions according to equations (7) and (8). Hence in the limit as $m$ increases the values converge to the continuously exerciseable American option price. ${ }^{23}$

The proof in Theorem 3 and the Corollary extend to the multivariate case, as shown for example in $\mathrm{He}$ (1990). Here, what is required again is that the option payoff is uniformly integral where the underlying state space is multivariate.

\section{An Example: The Valuation of a Twice Exerciseable, Quality Option}

Consider the following quality option contract. The option is to acquire either asset $X$ or asset $Y$. The holder has the additional option to exercise at time $t_{2}$, or time $t_{1}$. If he exercises at $t_{2}$, he can buy either asset $X$ at a price $K_{2, x}$ or asset $Y$ at a price $K_{2, y}$. Alternatively he can exercise his option at time $t_{1}$ and buy asset $X$ for $K_{1, x}$ or asset $Y$ for $K_{1, y}$. This is a quality option with an American feature. ${ }^{24}$

The payoffs on the option are as follows:

At time $t_{2}$,

$$
\begin{aligned}
g\left(X_{2}, Y_{2}\right) & =\max \left[X_{2}-K_{2, x}, Y_{2}-K_{2, y}, 0\right], & & \text { if option not exercised at } t_{1} \\
& =0, & & \text { if option exercised at } t_{1 .} .
\end{aligned}
$$

At time $t_{1}$ the value of the option is

$$
g\left(X_{1}, Y_{1}\right)=\max \left[X_{1}-K_{1, x}, Y_{1}-K_{1, y}, C_{1}\right],
$$


where $C_{1}$ is the value of the option at time $t_{1}$, if it is not exercised at time $t_{1}$. In order to value this option, we make the following assumptions: (1) Interest rates in the economy are non-stochastic, (2) $t_{1}=1$ and $t_{2}=2$, (3) The relevant prices of the assets $X_{1}, X_{2}, Y_{1}, Y_{2}$ are joint lognormally distributed, (4) A preference-free valuation relationship exists for the valuation of the option.

The fourth assumption implies that the option can be valued using a risk-neutral density function where the means of the stochastic variables are their respective forward prices. This implies that the means of the variables are

$$
\begin{array}{ll}
E\left(X_{1}\right)=F_{0,1, x}, & E\left(Y_{1}\right)=F_{0,1, y}, \\
E\left(X_{2}\right)=F_{0,2, x}, & E\left(Y_{2}\right)=F_{0,2, y},
\end{array}
$$

where $F_{0, t}$ refers to the forward price of the asset for delivery at time $t$. We approximate the required distributions with a binomial distribution which has $n$ stages from 0 to $t_{1}$ and from $t_{1}$ to $t_{2}$.

In Table 3, we show the input data for the option valuation. Note that the unconditional volatility for asset $X_{t}$ is less than its (constant) conditional volatility, indicating mean reversion. Also the conditional volatility of $Y_{t}$ is non-constant. For convenience we choose the forward prices of $X_{t}$ and $Y_{t}$ to be constant, equal to each other, and equal to 1 . The strike prices are also 1 at time $t_{2}$. At time $t_{1}$, the strike prices are assumed to be lower (0.96), in order to allow the possibility of early exercise, and make the valuation problem more interesting.

In Table 3, we illustrate the convergence of the option price as the number of binomial stages (both $n_{1}$ and $n_{2}$ for $X$ and $Y$ ) increases. Note that reasonably accurate answers are obtainable with $n=8$ even when the correlation between the variables is high. This is due to the fact that with 2 variables and 2 periods, there are $(n+1)^{4}$ states. Table 3 also shows that this quality option declines in value as the assumed correlation between the underlying assets increases: with $n=30$, the value is $8.85 \%$ in the uncorrelated case and only $7.06 \%$ when $\rho=0.8$. 
This example illustrates the rapid convergence of the method. Even for relatively small values of $n$ and highly correlated variables, the errors of the approximated process are relatively small. The resulting option prices are accurate and can be computed rapidly given the small values of $n$.

\section{Conclusions}

In this paper, we have shown that binomial distributions with changing probability parameters can be constructed to approximate the joint distribution of the asset price at various (exercise) dates. The method can be extended, using state dependent probabilities, to approximate the covariance characteristics in the multivariate case, where there is more than one state variable determining the option payoff. Tests of the accuracy of the multivariate binomial approximation for variables with changing variance and covariance characteristics show it to be quite accurate even with a small number of binomial stages. The accuracy also extends to cases where the correlation between the variables is high. The method offers a fast and efficient computational method for multiple-exerciseable options prices which can be extended to American-style option prices using the Geske-Johnson (1984) approximation technique. This would facilitate a generalization of the work of Breen (1991). Alternatively, it can be used to value a compound option whose payoff depends upon two or more variables.

The principal feature of our methodology is its computational efficiency compared with alternative option pricing methods using numerical integrations. This advantage is clear-cut for American options where exercise is possible on two or more dates, our approach is less computationally intensive. This is because in our method, the number of nodes increases only linearly with the number of exercise dates. For instance, numerical integration along the lines of Geske-Johnson (1984) would involve $n^{3}$ instead of $(3 n+1)$ nodes in our binomial method, in the thrice-exerciseable case. For options involving two or more state variables, and/or more exercise dates, the comparative efficiency of our methodology is even more significant. For example, if Amin's (1991) orthogonal factor method is used with three state variables, $(n+1)^{3}$ nodes are generated for the third variable whereas each variable has just $n+1$ nodes using our method. This leads to a dramatic 
reduction in the number of required integrations. For some options our method can make the difference between feasible and non-feasible computation. These computational advantages become even more important when hedge ratios and other risk management sensitivity parameters are required, in addition to the option values. 


\section{Appendix A: Proof of Lemma 1 and Theorem 2}

\section{Proof of Lemma 1}

(a) For a given $N_{i}$, the mean of the approximated process is:

$$
\frac{\hat{E}\left(X_{i}\right)}{X_{0}}=\prod_{l=1}^{i}\left[q\left(x_{l}\right) u_{i}+\left\{1-q\left(x_{l}\right)\right\} d_{i}\right]^{n_{l}} \text {. }
$$

Since, by the condition of the theorem, $q\left(x_{l}\right) \rightarrow 0.5$ as $n_{l} \rightarrow \infty, l=1,2, \ldots, i$,

$$
\lim _{\substack{n_{l} \rightarrow \infty \\ l=1,2, \ldots, i}} \frac{\hat{E}\left(X_{i}\right)}{X_{0}}=\left[0.5\left(u_{i}+d_{i}\right)\right]^{N_{i}} .
$$

From equation (8), however,

$$
u_{i}+d_{i}=2\left[\frac{E\left(X_{i}\right)}{X_{0}}\right]^{\frac{1}{N_{i}}}
$$

Hence,

$$
\lim _{\substack{n_{l} \rightarrow \infty \\ l=1,2, \ldots, i}} \frac{\hat{E}\left(X_{i}\right)}{X_{0}}=\frac{E\left(X_{i}\right)}{X_{0}}
$$

(b) The conditional variance can be written in the limit, since $q\left(x_{i}\right) \rightarrow 0.5$, when $n_{i} \rightarrow \infty$, ${ }^{25}$

as

$$
\begin{aligned}
\lim _{n_{i} \rightarrow \infty}\left[\hat{\sigma}_{i-1, i}\left(n_{i}\right)\right]^{2} & =\frac{1}{t_{i}-t_{i-1}} n_{i}(0.5)^{2}\left(\ln u_{i}-\ln d_{i}\right)^{2} \\
& =\frac{0.25 n_{i}}{t_{i}-t_{i-1}}\left[4 \sigma_{i-1, i}^{2} \frac{\left(t_{i}-t_{i-1}\right)}{n_{i}}\right]
\end{aligned}
$$

i.e. $\quad \lim _{n_{i} \rightarrow \infty}\left[\hat{\sigma}_{i-1, i}\left(n_{i}\right)\right]^{2}=\sigma_{i-1, i}^{2}$. 


\section{Proof of Theorem 2}

In the case of just two variables $(X, Y)$ it is sufficient to establish the theorem for the special case of $n_{x}=1$. For $n_{x}=1$, the means of the two variables $X$ and $Y$, where $Y$ is chosen to be conditional on $X$, are given by

$$
\begin{aligned}
& \mu_{x}=\left(\ln u_{x}-\ln d_{x}\right) / 2, \\
& \mu_{y}=q \ln u_{y}+(1-q) \ln d_{y}, \\
& q=\left(q_{0}+q_{1}\right) / 2,
\end{aligned}
$$

where $q_{r}=q\left(y \mid x=x_{r}\right)$ as defined by equation (11).

The conditional covariance between $X$ and $Y$ is given by

$$
\hat{\sigma}_{x y}=\left(\frac{1}{2}\right)^{2}\left(q_{0}-q_{1}\right)\left(\ln u_{x}-\ln d_{x}\right)\left(\ln u_{y}-\ln d_{y}\right) .
$$

But from (11), $q_{0}$ and $q_{1}$ are given by

$$
q_{r}=\frac{\alpha+\beta\left[r \ln d_{x}+(1-r) \ln u_{x}\right]-\ln d_{y}}{\ln u_{y}-\ln d_{y}}, \quad r=0,1
$$

so that the approximated covariance $\hat{\sigma}_{x, y}$ can be written as

$$
\hat{\sigma}_{x y}=\left(\frac{1}{2}\right)^{2} \beta\left(\ln u_{x}-\ln d_{x}\right)^{2}=\beta \sigma_{x}^{2}
$$

i.e. $\quad \hat{\sigma}_{x y}=\sigma_{x y}$.

A similar argument can be used for the case of three or more variables. For instance, consider the case of three variables $X, Y$, and $Z$, where $X$ is the first fitted variable. First, the proof for the case of two variables applies directly to the covariances between $X$ and $Y$, and $X$ and $Z$. Next, since in the limit, the binomial probability of $Y$ converges to 0.5 , then the covariance of $Z$ with $Y$ converges to its true value. 


\section{Appendix B: The Binomial Probability in the case of a Multivariate Lognormal Stochastic Process}

\section{The General Problem}

The method used in the paper can be applied to construct a binomial tree as a discrete-time approximation for any multivariate lognormal distribution. We first consider the general problem of approximating variables with a given covariance structure. We then apply this general method to the problem in the text. To see the specific details of the method consider the case of a trivariate lognormal distribution of 3 variables $X, Y$ and $Z$ with the following variance-covariance matrix (between the logarithms of the variables): ${ }^{26}$

$$
\Omega=\left(\begin{array}{ccc}
\sigma_{x}^{2} & \sigma_{x, y} & \sigma_{x, z} \\
\sigma_{y, x} & \sigma_{y}^{2} & \sigma_{y, z} \\
\sigma_{z, x} & \sigma_{z, y} & \sigma_{z}^{2}
\end{array}\right) .
$$

For notational convenience we use lower case letters to denote natural logarithms (i.e. $x=\ln X, y=\ln Y, z=\ln Z$ ).

Since $x, y$, and $z$ are normally distributed, the multiple regression

$$
\begin{aligned}
& z=\alpha_{z}+\beta_{z} x+\gamma_{z} y+\varepsilon, \\
& \beta_{z}=\frac{\sigma_{z, x} \sigma_{y}^{2}-\sigma_{y, z} \sigma_{x, y}}{\sigma_{x}^{2} \sigma_{y}^{2}-\sigma_{x, y}^{2}}, \\
& \gamma_{z}=\frac{\sigma_{y, z} \sigma_{x}^{2}-\sigma_{x, y} \sigma_{z, x}}{\sigma_{x}^{2} \sigma_{y}^{2}-\sigma_{x, y}^{2}}, \\
& \alpha_{z}=E(z)-\beta_{z} E(x)-\gamma_{z} E(y),
\end{aligned}
$$

where

and

is linear, and the conditional expectation of $z$ is

$$
E(z \mid x, y)=\alpha_{z}+\beta_{z} x+\gamma_{z} y .
$$


First, we construct separate binomial trees for the variable $x, y, z$, using the method described in the text. We then choose the conditional probability of an up movement in $z$ so that (B1) is satisfied at each node. Given that $z$ is a binomial process this implies that

$$
\alpha_{z}+\beta_{z} x_{r}+\gamma_{z} y_{s}=n_{z}\left\{q(z) \ln u_{z}+[1-q(z)] \ln d_{z}\right\},
$$

where $q(z)=q\left(z \mid x=x_{r}, y=y_{s}\right)$ is the probability of an up movement in $z$ given that $x$ is at node $r$ and $y$ is at node $s$ of their respective binomial distributions. In (B2) $n_{z}$ is the number of stages in the binomial process of $z$ and $u_{z}$ and $d_{z}$ are the up and down movements of $z$. Solving (B2) we find

$$
q(z)=\frac{\alpha_{z}+\beta_{z} x_{r}+\gamma_{z} y_{s}-n_{z} \ln d_{z}}{n_{z}\left(\ln u_{z}-\ln d_{z}\right)} .
$$

\section{The Time Series Problem with Two Time Periods}

In the text we consider the problem of constructing a process for $X_{t}$ with given volatility characteristics at two specified points in time $t_{i-1}$ and $t_{i}$. In this case we construct binomial trees of $z=x_{i}$ and $x=x_{i-1}$. The resulting values of $X_{i-1}$ and $X_{i}$ are illustrated in Figure 1 in the text. The difference in this case is that $x_{i}$ is a time-series (i.e. a cumulative) variable. In this case, the conditional expectation of $x_{i}$ given node $r$ at time $t_{i-1}$ is

$$
a_{i}+b_{i} x_{i-1}=n_{i}\left\{q\left(x_{i}\right) \ln u_{i}+\left[1-q\left(x_{i}\right)\right] \ln d_{i}\right\}+\left(N_{i-1}-r\right) \ln u_{i}+r \ln d_{i} .
$$

In (B4) the first term represents the drift from time $t_{i-1}$ to time $t_{i}$. The second term is the expected value of $x_{i}$, given that the variable is in state $r$ at time $t_{i-1}$, if the drift from time $t_{i-1}$ to $t_{i}$ is zero. ${ }^{27}$

Solving (B4) for $q\left(x_{i}\right)$ we have in this case

$$
q\left(x_{i}\right)=\frac{\alpha_{i}+\beta_{i} x_{i-1}-\left[\left(N_{i-1}-r\right) \ln u_{i}+r \ln d_{i}\right]-n_{i} \ln d_{i}}{n_{i}\left(\ln u_{i}-\ln d_{i}\right)},
$$

where $q\left(x_{i}\right)=q\left(x_{i} \mid x_{i-1}=x_{i-1, r}\right)$ is the probability of an up movement in $x_{i}$ given that $x_{i-1}$ is at node $r$. 


\section{The Multivariate Time Series Problem}

In the text we consider the example where there are two relevant variables $\left(X_{t}, Y_{t}\right)$ and two time periods $(i-1, i)$. In this case we suggest first constructing the binomial process for $\left(X_{i-1}, X_{i}\right)$ and the relevant conditional probabilities $q\left(x_{i}\right)$ using the methods described above. The remaining problem is to compute the conditional probability of $Y_{2}$ given both $Y_{1}$ and $X_{2}$. This must reflect both the time series properties of the $Y_{t}$ process and the conditional correlation of the two variables $\left(\rho_{1,2, x, y}\right)$.

This is a multivariate problem involving 3 variables $\left(Y_{i}, Y_{i-1}, X_{i}\right)$. Hence we can use the general case in Section 1 of Appendix B above with $z=Y_{i}, x=Y_{i-1}$, and $y=X_{i}$. Again we recognize that $y_{i}$ is a time-series variable. In this case therefore, the conditional expectation of $y_{i}$, given node $r$ of $y_{i-1}$ and node $s$ of $x_{i}$ is $a_{i}+b_{i} y_{i-1}+c_{i} x_{i}=n_{i}\left\{q\left(y_{i}\right) \ln u_{i, y}+\left[1-q\left(y_{i}\right)\right] \ln d_{i, y}\right\}+\left(N_{i-1}-r\right) \ln u_{i, y}+r \ln d_{i, y}$.

Solving (B5) for $q\left(y_{i}\right)$ we have

$$
q\left(y_{i}\right)=\frac{a_{i}+b_{i} y_{i-1}+c_{i} x_{i}-\left[\left(N_{i-1}-r\right) \ln u_{i, y}+r \ln d_{i, y}\right]-n_{i} \ln d_{i, y}}{n_{i}\left(\ln u_{i, y}-\ln d_{i, y}\right)},
$$

where $q\left(y_{i}\right)=q\left(y_{i} \mid y_{i-1}=y_{i-1, r}, x_{i_{i}}=x_{t_{i}, s}\right)$ is the conditional probability of an up movement in $y_{i}$ given that $y_{i-1}$ is at node $r$ and $x_{i}$ is at node $s$. 


\section{Footnotes}

1. In the case of many path-dependent options the problem, however, is that the number of paths explodes with the number of stages in the binomial tree. Hence such options can only be valued for quite small $n$-size binomial trees.

2. Other examples of multivariate options include the "delivery option" that is embedded in many bond futures contracts, a "cocktail" option to receive the principal payment on a bond in one of many currencies at specified exchange rates, and a long-term American-style currency option under stochastic interest rates. For other examples, see Stulz (1982) and Boyle, Evnine and Gibbs (1989).

3. Note that a term structure of volatility can result either from mean reversion, changing conditional volatility or both.

4. For example, if, as in Breen (1991), we are approximating an American option using the Geske-Johnson (1984) technique, using a trivariate distribution, we would be interested in three dates, $t_{1}, t_{2}, T$.

5. Note that, given our assumptions, the volatilities and covariances are state independent.

6. There are, however, natural restrictions that have to be imposed on the variance-covariance matrices. In particular, the relevant variance-covariance matrices have to be positive semi-definite.

7. Note that it is not restrictive in any sense to confine our attention to the series of dates $t_{1}, t_{2}, \ldots, t_{i}, \ldots, t_{m}$, since the time intervals between the dates could be made as small as desired.

8. An alternative method when the correlation between the variables is high is to orthogonalize the variables and then construct the binomial tree.

9. A method similar to that in Nelson and Ramaswamy was suggested and implemented in Stapleton and Subrahmanyam (1988).

10. We drop the subscript $j$ in this Section. For instance, instead of $\sigma_{0, i, j}$ and $\sigma_{i-1, i, j}$ we simply write $\sigma_{0, i}$ and $\sigma_{i-1, i}$ respectively. Also instead of $X_{i, j}$ we simply write $X_{i}$.

11. Note that the convergence of the approximated mean to the true mean implies that the approximated logarithmic mean $\hat{\mu}_{i}$ also converges to the true logarithmic $\mu_{i}$.

12. Also, since $X_{i}$ is lognormally distributed, $E\left(x_{i}\right)=\ln \left(\frac{E\left(X_{i}\right)}{X_{0}}\right)-\frac{1}{2} \sigma_{0, i}^{2}$.

13. Note that (9) has to hold for the approximated process, while it is true by assumption for the original variables.

14. Note that the proof in the Appendix B refers to the trivariate case with coefficients $\alpha, \beta$ and $\gamma$.

15. Note that this is always a "small $n$ " problem, since $q\left(x_{2}\right)$ in (10) limits to 0.5 as $n_{i} \rightarrow \infty$. This can be seen from (10) where the second term goes rapidly to 0.5 as $n_{i}$ increases and the first term can be kept within the range -0.5 to 0.5 by choosing a large enough size of $n_{i}$.

16. Simulations of the case where $X_{i}$ is fitted over 3 periods $t_{1}, t_{2}, t_{3}$ yield similar results. For example, assuming constant conditional volatility of $10 \%$ and mean reversion such that $\sigma_{0,2}=0.09, \sigma_{0,3}=0.08$, we found that for $n=10, \hat{\sigma}_{0,3}=0.07974(26) ; n=30, \hat{\sigma}_{0,3}=0.07991(9) ; n=50, \hat{\sigma}_{0,3}=0.07995(5)$. Similar results were obtained for the case of changing volatility, and where the process has both mean reversion and volatility change.

17. Boyle (1988) develops a technique for the multivariate case based on a trinomial distribution. Our method differs from Boyle's in that we use a simpler binomial distribution. Our method allows us to solve the general problem of constructing a multivariate process with arbitrary cross-sectional variances and covariances, and conditional variances and covariances over time. 
18. An alternative method suggested by Amin (1991) models the covariance using factor loadings on two independent random variables. A problem with this approach is that the number of $Y$ and $Z$ nodes increases rapidly with the $n$-size making the computation of complex option prices difficult.

19. This follows from equation (B3) with $z=x$ and $\gamma_{z}=0$.

20. In fact the inaccuracy can be eliminated by appropriate choice of $n$-size for the dependent variable.

21. The prices which our finite $n$-size economy values converge to are thus the no-arbitrage prices. Our method is in the spirit of Rajasingham (1990) who shows that it is not necessary for the finite economies to be complete when the economies are used as approximations. Our task, therefore, differs somewhat from that of Cox, Ross, and Rubinstein (1979) and Duffie (1988) who assume knowledge of the asset's true mean and show that the no-arbitrage, finite economy prices converge to the continuous economy limit. It is interesting to note that $\mathrm{He}(1990)$ and Amin (1990) both suggest an alternative three-state approach to the valuation of options on multivariate processes which allows a complete market, no-arbitrage, valuation in the finite economy. In our bivariate binomial case, the finite economy is incomplete; however, our approximated values can still be shown to converge to the continuous-time limiting values.

22. This follows from Geske and Johnson (1984).

23. Strictly, this argument requires that the strike price of the option is a continuous function of time. Otherwise, it is possible for example, that the option payoff could be positive if exercised at an irrational date and zero otherwise. An alternative rigorous proof of convergence for American options is provided by Amin and Khanna (1994).

24. An example of a quality option is the option available in most bond futures contracts. When we use the term "American" feature we mean that it can be exercised (at least once) before the final maturity.

25. See Cox and Rubinstein (1985), p. 201.

26. The method is readily generalized to the $n$-variable case. However, the notation is complex.

27. Note that, in the special case where the volatility is constant over time $u_{z}=u_{x}$ and (B4) becomes

$$
a_{i}+b_{i} x_{i-1}=n_{i}\left\{q\left(x_{i}\right) \ln u_{i}+\left[1-q\left(x_{i}\right)\right] \ln d_{i}\right\}+x_{i-1} .
$$

In this case the conditional expectation of $x_{i}$ is the drift plus $x_{i-1}$. 


\section{References}

Amin, K.I. (1990), “A Simplified Discrete Time Approach for the Pricing of Derivative Securities with Stochastic Interest Rates," Working Paper, School of Business Administration, University of Michigan, Ann Arbor.

Amin, K.I. (1991), "On the Computation of Continuous Time Option Prices Using Discrete Approximations,' Journal of Financial and Quantitative Analysis, 26, 477-495.

Amin, K.I. and J.N. Bodurtha, Jr. (1994), "Pricing American Options with Stochastic Interest Rates: International Money Market Applications", Review of Financial Studies, forthcoming.

Amin, K.I. and A. Khanna (1994), "Convergence of American Option Values from Discrete to Continuous-time Financial Models," Mathematical Finance, forthcoming.

Billingsley, P. (1986), Probability and Measure, 2nd Edition, John Wiley and Sons, New York.

Breen, R. (1991), "The Accelerated Binomial Option Pricing Model," Journal of Financial and Quantitative Analysis, 26, 153-164.

Boyle,P.P.(1988), “A Lattice Framework for Options Pricing when there are Two State Variables,” Journal of Financial and Quantitative Analysis, 23, 1-12.

Boyle, P.P., J. Evnine and S. Gibbs (1989), "Numerical Evaluation of Multivariate Contingent Claims," Review of Financial Studies, 2, 241-250.

Bunch, D.S. and H. Johnson (1992), “A Simple and Numerically Efficient Valuation Method for American Puts Using a Modified Geske-Johnson Approach," Journal of Finance, 47, 809-816.

Cox, J.C. and M. Rubinstein (1985), Options Markets, Prentice-Hall, Englewood Cliffs, New Jersey.

Cox, J.C., S.A. Ross and M. Rubinstein (1979), “Option Pricing: A Simplified Approach," Journal of Financial Economics, 7, 229-263.

Duffie, D. (1988), Security Markets, Academic Press, Inc., San Diego, California.

Geske, R. (1979), “'The Valuation of Compound Options,”' Journal of Financial Economics, 7, 63-81.

Geske, R. and H. Johnson (1984), "The American Put Valued Analytically," Journal of Finance, 39, 1511-1542.

He, H. (1990), "Convergence from Discrete- to Continuous-Time Contingent Claims Prices," Review of Financial Studies, 3, 523-546.

Nelson, D.B. and K. Ramaswamy (1990), "Simple Binomial Processes as Diffusion Approximations in Financial Models," Review of Financial Studies, 3, 393-430.

Rajasingham, A. (1990), “Convergence of Discrete Time Event Trees to Continuous Time Economies and the Pricing of Contingent Claims," Technical Report, International Monetary Fund, Washington DC.

Stapleton, R.C. and M.G. Subrahmanyam (1988), “The Valuation of Options on Portfolios," Proceedings of the 15th Annual Conference of the European Finance Association, Madrid, Spain.

Stulz, R.M. (1982), "Options on the Minimum or the Maximum of Two Risky Assets: Analysis and Applications," Journal of Financial Economics, 10, 161-185. 


\section{Table 1}

Convergence of Binomial Approximation in a two period example with (1) Mean Reversion, (2) Changing Volatilities, and (3) Mean Reversion and Changing Volatilities

\begin{tabular}{|c|c|c|c|c|c|}
\hline \multicolumn{3}{|c|}{ (1) Mean Reversion ${ }^{\mathfrak{c}}$} & \multicolumn{3}{|c|}{ Volatility of Approximated Process, $\hat{\sigma}_{0,2}{ }^{\mathrm{a}}$} \\
\hline$\sigma_{0,1}$ & $\sigma_{1,2}$ & $\sigma_{0,2}$ & $n=10$ & $n=30$ & $n=50^{\mathrm{b}}$ \\
\hline 0.10 & 0.10 & 0.10 & 0.10000 & 0.10000 & 0.10000 \\
\hline 0.10 & 0.10 & 0.09 & $0.08987(13)^{\mathrm{f}}$ & $0.08996(4)$ & $0.08997(3)$ \\
\hline 0.10 & 0.10 & 0.08 & $0.07930(70)$ & $0.07977(23)$ & $0.07986(14)$ \\
\hline \multicolumn{3}{|c|}{ (2) Changing Volatilities ${ }^{d}$} & \multicolumn{3}{|c|}{ Volatility of Approximated Process, $\hat{\sigma}_{0,2}$} \\
\hline$\sigma_{0,1}$ & $\sigma_{1,2}$ & $\sigma_{0,2}$ & $n=10$ & $n=30$ & $n=50$ \\
\hline 0.10 & 0.09 & 0.09513 & $0.09510(3)$ & $0.09512(1)$ & $0.09513(0)$ \\
\hline 0.10 & 0.08 & 0.09055 & $0.09044(11)$ & $0.09051(4)$ & $0.09053(2)$ \\
\hline 0.10 & 0.07 & 0.08631 & $0.08605(26)$ & $0.08622(9)$ & $0.08626(5)$ \\
\hline \multicolumn{3}{|c|}{$\begin{array}{l}\text { (3) Mean Reversion and } \\
\text { Changing Volatilities }\end{array}$} & \multicolumn{3}{|c|}{ Volatility of Approximated Process, $\hat{\sigma}_{0,2}{ }^{2}$} \\
\hline$\sigma_{0,1}$ & $\sigma_{1,2}$ & $\sigma_{0,2}$ & $n=10$ & $n=30$ & $n=50$ \\
\hline 0.10 & 0.09 & 0.08513 & $0.08510(3)$ & $0.08512(1)$ & $0.08513(0)$ \\
\hline 0.10 & 0.08 & 0.08055 & $0.08055(0)$ & $0.08055(0)$ & $0.08055(0)$ \\
\hline 0.10 & 0.07 & 0.07631 & $0.07626(5)$ & $0.07629(2)$ & $0.07630(1)$ \\
\hline
\end{tabular}

Notes:

a. In each case the periods are $t_{1}=1$ year, $t_{2}=2$ year.

b. In all cases $n$ is the same for year 1 and year 2, i.e. $n_{1}=n, n_{2}=n$.

c. All volatilities are annualized. The volatility over the first period $\sigma_{0,1}$ is exact, by construction.

d. In this case we assume that $X_{t}$ follows a random walk with changing variance. Hence $\sigma_{0,2}^{2}=\left(\sigma_{0,1}^{2}+\sigma_{1,2}^{2}\right) / 2$ in this case.

e. In this case we assume that $X_{t}$ follows a mean reverting process with changing conditional variance. In these examples, we choose $\sigma_{0,2}=\sqrt{\left(\sigma_{0,1}^{2}+\sigma_{1,2}^{2}\right) / 2}-0.01$.

f. In brackets we show $\left(\sigma_{0,2}-\hat{\sigma}_{0,2}\right) \times 100,000$. This is the approximation error. This is also the size of the error $\left(\sigma_{1,2}-\hat{\sigma}_{1,2}\right)$. 


\section{Table 2}

Accuracy of the Binomial Approximation in a Multivariate Case With and Without Non-Negativity Constraint on Probabilities

\begin{tabular}{ccccc}
\hline & \multicolumn{3}{c}{ Volatility of Approximated Process, $\hat{\sigma}_{0,1, y}$} \\
& \multicolumn{2}{c}{ Binomial Stages, } & \multicolumn{2}{c}{ Binomial Stages, } \\
& $n_{1, x}=n_{1, y}=10$ & $n_{1, x}=n_{1, y}=20$ \\
Correlation, & Estimated & Approximation & Estimated & Approximation \\
\cline { 2 - 5 }$\rho_{0,1, x, y}$ & $\hat{\sigma}_{0,1, y}$ & Error & $\hat{\sigma}_{0,1, y}$ & Error \\
\hline 0.0 & 0.1000 & 0 & 0.1000 & 0 \\
0.1 & 0.0999 & 1 & 0.1000 & 0 \\
0.2 & 0.0998 & 2 & 0.0999 & 1 \\
0.3 & 0.0995 & 5 & 0.0998 & 2 \\
0.4 & 0.0992 & 8 & 0.0986 & 4 \\
0.5 & 0.0987 & 13 & 0.0984 & 6 \\
0.6 & 0.0982 & 18 & 0.0991 & 9 \\
0.7 & 0.0975 & 25 & 0.0988 & 12 \\
0.8 & 0.0960 & 40 & 0.0984 & 16 \\
$0.8^{\text {d }}$ & $(0.0967)$ & $(33)$ & $(0.0984)$ & $(16)$ \\
0.9 & 0.0878 & 122 & 0.0961 & 39 \\
$0.9^{\text {d }}$ & $(0.0959)$ & $(41)$ & $(0.0979)$ & $(21)$ \\
\hline
\end{tabular}

Notes:

a. In all cases, each of the volatilities (conditional and unconditional) for $X$ and $Y$ is $10 \%$.

b. $t_{1}=1$ year.

c. The approximation error is shown $\left(\sigma_{0,1, y}-\hat{\sigma}_{0,1, y}\right) \times 100,000$.

d. The number in brackets below the constrained estimated volatility $\hat{\sigma}_{0,1, y}$ is the corresponding unconstrained volatility estimate. 
Table 3

A Twice Exerciseable Option: Sensitivity Analysis

\begin{tabular}{cccccc}
\hline Correlation & \multicolumn{5}{c}{ Number of Binomial Stages ${ }^{\mathrm{b}}, n$} \\
\cline { 2 - 6 } Coefficient $^{\mathrm{a}}, \rho$ & 4 & 8 & 12 & 16 & 30 \\
\hline 0.0 & 0.0881 & 0.0884 & 0.0884 & 0.0885 & 0.0885 \\
0.2 & 0.0845 & 0.0849 & 0.0850 & 0.0850 & 0.0849 \\
0.4 & 0.0802 & 0.0808 & 0.0810 & 0.0810 & 0.0810 \\
0.6 & 0.0750 & 0.0759 & 0.0761 & 0.0763 & 0.0764 \\
0.8 & 0.0677 & 0.0694 & 0.0700 & 0.0702 & 0.0706 \\
\hline
\end{tabular}

Notes:

a. This is the value of $\rho$ both for time $t_{1}$ and for time $t_{1}$ to $t_{2}$.

b. The $n$ size applies to $n_{1}$ for $X$ and $Y$, and to $n_{2}$ for both $X$ and $Y$.

c. The following input data for the twice exerciseable quality option on two assets is assumed for all option price calculations:

\begin{tabular}{lll}
\hline Time & $t_{1}=1$ year & $t_{2}=2$ years \\
Forward Prices $^{\mathrm{d}}$ & $F_{0,1, x}=F_{0,1, y}=1$ & $F_{0,2, x}=F_{0,2, y}=1$ \\
Zero Bond Prices $^{\mathrm{e}}$ & $B_{0,1}=0.9$ & $B_{0,2}=0.81$ \\
Volatility: & & \\
$\quad \sigma_{0,1, x}=0.1$ & $\sigma_{1,2, x}=0.1$ \\
$\quad$ Conditional $(x)$ & $\sigma_{0,1, y}=0.08$ & $\sigma_{1,2, y}=0.07$ \\
$\quad$ Unconditional $(y)^{\text {Unconditional }}{ }^{\mathrm{g}}(x)$ & & $\sigma_{0,2, x}=0.09$ \\
$\quad$ & & $\sigma_{0,2, y}=0.07517$ \\
Strike Prices & $K_{1, x}=K_{1, y}=0.96$ & $K_{2, x}=K_{2, y}=1$ \\
\hline
\end{tabular}

d. The fact that forward prices are the same for delivery at years 1 and 2 implies that the assets pay dividends.

e. These prices imply a constant rate of interest of $11.11 \%$ per year.

f. These volatilities are all quoted on an annualized basis.

g. This lower unconditional volatility implies mean reversion in the $X_{t}$ process.

h. $\quad \sigma_{0,2, y}=\sqrt{\left(\sigma_{0,1, y}^{2}+\sigma_{1,2, y}^{2}\right) / 2}$.

Hence $y$ follows a random walk with changing variance. 


\section{Figure 1}

A Discrete Process for $X_{1}, X_{2}$

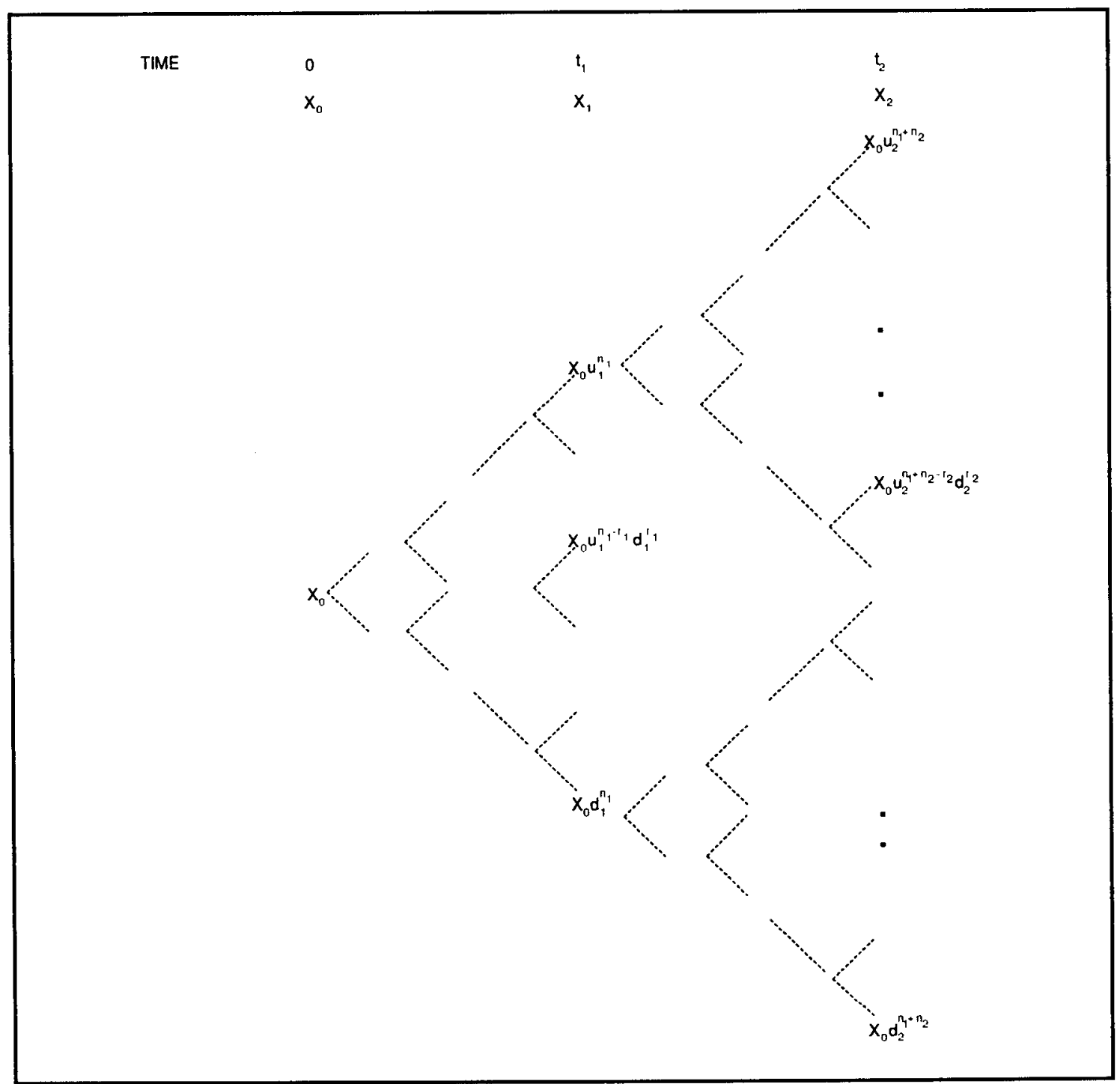

There are $n_{1}+1$ nodes at $t_{1}$ numbered $r=0,1, \ldots, n_{1}$. There are $n_{1}+n_{2}+1$ nodes at $t_{2}$ numbered $r=0,1, \ldots, n_{1}+n_{2}, X_{0}$ is the starting price, $X_{1}$ is the price at time $t_{1}, X_{2}$ is the price at time $t_{2} . u_{1}, d_{1}, u_{2}$, and $d_{2}$ are the proportionate up and down movements. Although $X_{1}$ and $X_{2}$ are generated by binomial processes, this method gives two vectors $\left(n_{1}+1\right.$ dimensional for $X_{1}$ and $n_{2}+n_{1}+1$ dimensional for $\left.X_{2}\right)$. Intermediate values at time $t \in\left(0, t_{1}\right), t \in\left(t_{1}, t_{2}\right)$ are not defined. 